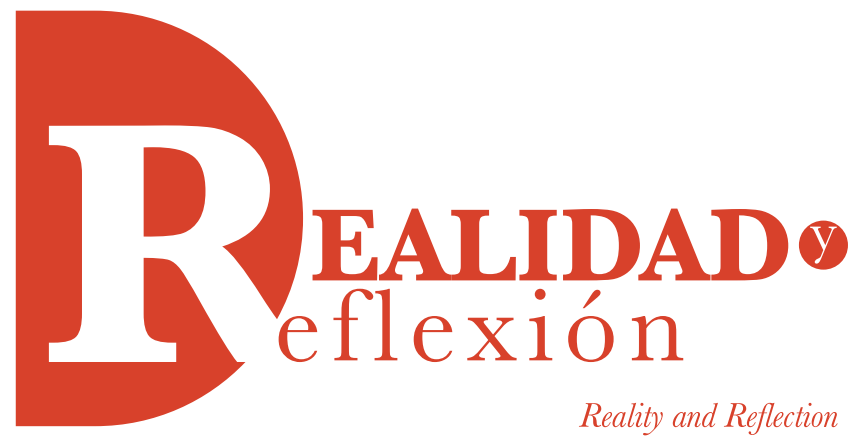

ISSN 1992-6510

e-ISSN 2520-92990

Año 17, No 45, San Salvador, El Salvador, Centroamérica. Revista Semestral Enero-Junio 2017

YEAR 17, No 45, SAN SALVADOR, EL SALVADOR, CENTRAL AMERICA. SEMESTRAL JOURNAL JANUARY-JUNE 2017

\title{
Evaluación de la estrategia educativa «Todos Pueden Aprender» desde la perspectiva del profesorado
}

\author{
Evaluation of the educational strategy «Everyone Can \\ Learn» from the perspective of teachers
}

\author{
Adán Mendoza \\ Licenciado en Sociología por la Universidad de E1 Salvador. \\ Candidato a Magister en Docencia Universitaria por la Universidad de Oriente. \\ Profesor e investigador en la Dirección de Investigación de la Universidad de Oriente, El Salvador. \\ arivas@univo.edu.sv
}

Fecha de recepción: 18 de abril de 2017 Fecha de aceptación: 19 de mayo de 2017

\section{RESUMEN}

La presente investigación es de tipo evaluativo con enfoque cualitativo, y tuvo como objetivo, precisamente, evaluar desde las representaciones de los docentes el proceso de implementación de la estrategia educativa “Todos Pueden Aprender". Dicha estrategia se ha implementado en El Salvador desde el año 2012 por parte del Ministerio de Educación, con la finalidad de disminuir los niveles de fracaso escolar en el primer ciclo de educación básica. Así se estudiaron cuatro Centros Escolares de la ciudad de San Miguel, empleando entrevistas a profundidad a los docentes. Los hallazgos indican que desde la perspectiva de los profesores la implementación de la estrategia educativa ha presentado sostenibilidad en su ejecución, pero con problemas en la organización institucional (encuadre normativo), en los recursos didácticos para la aplicación de las secuencias didácticas en el aula y de consenso para el seguimiento de los docentes, de la metodología de la promoción asistida.

Palabras clave: Todos Pueden Aprender, secuencias didácticas, promoción asistida, fracaso escolar.

\section{ABSTRACT}

The present research is of an evaluative type with a qualitative focus, and it aimed, precisely, to gauge from the teachers perspective the process of implementation of the educational strategy "Everyone Can Learn" (Todos Pueden Aprender). This strategy has been implemented in El Salvador since 2012 by the Ministry of Education, with the purpose of diminishing the levels of school failure In the first cycle of basic education. To collect data, this research administered interviews with the teachers of fours schools in the city of San Miguel. The findings indicate that from the perspective of teachers, the implementation of the educational strategy has presented sustainability in its execution, but with problems in the institutional organization (normative framework), in the resources for the application of the didactic sequences in the classroom and the consensus for the follow-up of the methodology of assisted promotion.

Keywords: Everyone Can Learn, didactic sequences, assisted promotion, school failure. 


\section{Introducción}

La educación es uno de los pilares importantes para el desarrollo social. En ella, estriba la responsabilidad de la formación de los seres humanos con capacidades idóneas para aportar significativamente al progreso y el bienestar en general de la sociedad. En El Salvador, se ha reconocido a nivel "formal" la necesidad de fortalecer las oportunidades de desarrollo a través de la cualificación de la educación para todos y todas, con especial interés en los primeros años de formación. No obstante, aún existen diferencias significativas en el acceso a una educación de calidad, derivadas de las desigualdades socioeconómicas imperantes. Verbigracia, la UNESCO (2014) devela que en el país a nivel de educación básica "el 42\% de los niños de los hogares más pobres finalizan la enseñanza primaria y dominan las competencias básicas, frente al $84 \%$ de los provenientes de los hogares más ricos”.

Ciertamente, el rendimiento escolar de los estudiantes de los primeros años de educación básica es una preocupación frecuente en los entornos caracterizados por la pobreza y la vulnerabilidad social. Principalmente, porque aún persisten niveles significativos de fracaso escolar, evidenciados en la dificultad de los niños para el aprendizaje de la lectoescritura y el dominio sobre las operaciones elementales de las matemáticas, derivando gradualmente esta situación en potenciar el surgimiento de otros problemas educativos tales como: la repitencia, el ausentismo, sobre-edad y el abandono escolar temprano. Según el censo matricular del Ministerio de Educación (MINED) (2010), “en el año 2008 hubo 85,438 estudiantes que repitieron grado, de los cuales el
$71 \%$ se encuentra en los primeros dos ciclos de educación básica (p.12)”.

Como respuesta a esta situación, el MINED ha implementado en el país un conjunto de planes y programas cuyo fin ha sido la mejora significativa en el acceso a la educación básica, con énfasis en el desarrollo paulatino de la calidad, con resultados satisfactorios en términos de la ampliación a la cobertura, pero exiguos en lo que respecta a elevar el logros de los aprendizajes en las materias esenciales del proceso educativo básico. En el informe "Educación para todos" presentado a la UNESCO por parte del MINED en 2014, se destaca que la tasa de terminación de estudios primarios por cohorte paso de ser en promedio para el año 2000 de un $63.73 \%$ a un $76.54 \%$ hasta el año 2011. Pero, al observar los resultados en el logro de aprendizajes para ambos sexos en el área de matemáticas para tercer grado fueron para el año 2008 de apenas 5.40, y para el área de Lenguaje y Literatura, para el mismo grado, el promedio fue de 5.48. Entre las razones de esta situación, no se puede dejar de considerar el fracaso escolar en los primeros años.

En este contexto, una de las estrategias educativas a la cual se le ha apostado por parte de las autoridades del sistema educativo público para mejorar la calidad de los procesos de enseñanzaaprendizaje es la denominada: "Todos Pueden Aprender” (TPA), diseñada para aplicarse en el primer ciclo de educación básica. Dicha iniciativa es respaldada por la Coordinación Educativa y Cultural Centroamericana y el Sistema de Integración Centroamericana (CECC/SICA). Se lanzó en el marco del "Plan Social Educativo" y la ejecución de la Política de Educación Inclusiva, en la que se ha tomado a bien la aplicación de 
la mencionada estrategia como instrumento del Programa "Escuela Inclusiva de Tiempo Pleno", desde el año 2012. En un inicio se ejecutó la TPA con la capacitación a más de 300 docentes, directores y asesores pedagógicos con el objetivo de profundizar en los conceptos y las tareas claves para favorecer la enseñanza, el aprendizaje y el acompañamiento de los alumnos en las escuelas durante sus primeros años de estudio.

\section{Descripción de la estrategia educativa "TPA"}

La TPA es una estrategia educativa con cuna en Argentina, donde ha alcanzado el grado de política integral de cuidado a la infancia y ha tenido aplicabilidad jurisdiccional en las provincias de Chaco, Misiones, Tucumán, Jujuy, Santa Fe, Mendoza, Entre Ríos, San Juan y Formosa (Asociación Civil Educación para Todos, 2015). Fue diseñada por la "Asociación Civil Educación para Todos" con el auspicio y financiamiento del Fondo para la Naciones Unidas para la Infancia, o por sus siglas en inglés, UNICEF. Propone toda una estrategia didáctica y organizativa que pretende superar el fracaso escolar en el primer ciclo de educación básica.

En el documento de trabajo denominado: "Propuestas para superar el fracaso escolar" (2007) se ofrece una perspectiva de las razones pedagógicas y sociales que dan lugar a su formulación en la Argentina, pero que son perfectamente extrapolables a la realidad educativa salvadoreña:

En la actualidad, muchos son los niños y niñas que tienen vulnerado el pleno ejercicio del derecho a una educación de calidad. Entre las razones no podemos dejar de destacar, como un fuerte condicionante, al fracaso escolar en los primeros grados. Estas primeras experiencias negativas suelen condenar a la infancia a una situación en la que el acceso a otros derechos y por ende, a mejores oportunidades de desarrollo personal y social, se vuelve problemático. E1 fracaso perjudica la consolidación de la autoestima y reduce la confianza en su capacidad para enfrentar situaciones desafiantes. Por otro lado, la repitencia en los primeros años predice el doble de probabilidades de abandonar la escuela secundaria sin completarla. Estos costos son desiguales, pues afectan mayoritariamente a los niños y niñas más pobres (p.7).

En efecto, la estrategia es una propuesta de intervención didáctica que tiene como finalidad el incremento de las oportunidades de aprendizaje para todos los niños y niñas, que tradicionalmente son obligados a repetir el grado por motivos pedagógicos difusos o poco fundamentados, más allá de no haber aprendido a leer y escribir. Es debido a esta situación que la estrategia contempla dos propósitos esenciales: aumentar la cantidad de estudiantes que dominan los contenidos en las áreas de lenguaje y matemáticas que son inherentes en la currícula del primer ciclo de educación básica; y, reducir la cantidad de alumnos que experimentan fracasos escolares, en tanto, dicho fracaso sirve como motor al surgimiento de otros problemas educativos, previamente mencionados. (UNICEF, 2007).

Para aplicar la estrategia educativa TPA se requiere el trabajo de equipo entre directores, 
asesores y maestros de las instituciones educativas con base en a la metodología de la promoción asistida, entendida como alternativa a la repitencia, y la ejecución de secuencias didácticas innovadoras adecuadas a entornos difíciles. Se centra en este sentido, en la mejora de las prácticas pedagógicas de los docentes para la enseñanza de la lengua (alfabetización inicial) y las matemáticas.

\section{Metodología}

El objeto de estudio fue abordado bajo el tipo de investigación evaluativa, con enfoque cualitativo. La evaluación en investigación ha sido definida por Stufflemean como "el proceso de diseñar, obtener y proporcionar información útil para juzgar alternativas de solución" (como citó Bausela, 2004. p.185). Y, dentro del amplio paradigma cualitativo o naturalista, es el "enfoque interpretativo" (González Moteagudo, 2001) la corriente epistemológica que más se acomoda a los objetivos de la presente investigación. Para la interpretación del texto se utilizó el método hermenéutico-dialéctico que es definido por Teppa como: "la disciplina de la interpretación; con este método se trata de comprender textos y fenómenos socioeducativos. Se puede conocer el pensamiento humano con la ayuda de símbolos y palabras analizados en su contexto y realidad sociohistórica" (como citó Gasperi Romero, 2010, p. 81).

El universo que cubre la aplicación de la estrategia educativa TPA en la zona oriental de E1 Salvador es alrededor de 60 escuelas, y específicamente en el municipio de San Miguel están registradas un total de 10 escuelas piloto. No obstante, algunas escuelas presentan diferencias importantes en torno a la ejecución de la estrategia, por lo que, para la evaluación se seleccionaron las escuelas en relación al cumplimiento de los siguientes criterios:

a. Estudiar aquellos centros escolares en los que su personal hubiese recibido la primera capacitación y asistencia técnica por parte del MINED. Esto les permitirá tener representaciones del proceso de inducción y una importante experiencia acumulada en torno a la aplicación de la metodología y la gestión escolar.

b. La sostenibilidad de la aplicación de la metodología desde la fase de su desarrollo inicial hasta la fecha de realización de la evaluación. Esto permitirá extraer representaciones relativas a la dificultades y facilidades, logros y aprendizajes, etc.

c. Las características de las escuelas debían presentar deficiencias de recursos. Una escuela con alta inversión de recursos facilita la aplicación de la estrategia, el interés era evaluar aquellas con mayor dificultad de acceso a recursos didácticos y financieros.

d. Todas debían de pertenecer al municipio de San Miguel.

En este sentido, se decidió realizar las entrevistas en los siguientes centros escolares de la ciudad de San Miguel. (Ver Tabla n. ${ }^{\circ}$ ).

Finalmente, se aplicaron las técnicas e instrumentos de recolección de la información a ocho personas, las cuales estuvieron distribuidas en dos grupos: un primer grupo conformado por cuatro profesores, y otro por cuatro directores. 
Tabla n. ${ }^{\circ} 1$

Centros escolares seleccionados

\begin{tabular}{c|c|c|c}
\hline n. $^{\circ}$ & Nombre del centro escolar & Cargos & Cantidad \\
\hline 1 & Centro Escolar “Colonia La Presita” & Director y Profesor & 2 \\
\hline 2 & Centro Escolar “Abdón Cordero" & Director y Profesor & 2 \\
\hline 3 & Centro Escolar “Dolores C. Retes” & Director y Profesor & 2 \\
\hline 4 & Centro Escolar “Sagrado Corazón” & Director y Profesor & 2 \\
\hline \multicolumn{4}{r}{} \\
\hline
\end{tabular}

Algunas de las características de las personas participantes son: el total de los entrevistados/as (docentes y directores) sobrepasan los 50 años de edad, siendo las 4 docentes mujeres, y los directores tres hombres y una mujer. Todos son docentes con plaza fijas por ley de salarios. El nivel educativo de las docentes y los/as directores era de licenciatura, a excepción de una docente con nivel de profesorado.

\section{Análisis e interpretación de los resultados}

Para el análisis e interpretación de los datos cualitativos se leyeron varias veces las trascripciones y se tomaron notas para detectar regularidades y patrones. Posteriormente se procedió a la clasificación de toda la información escogiendo tres categorías. En cada una de ellas se segregaron una serie de subcategorías que responden a la información aportada por los distintos informantes y respaldada en la revisión de los documentos oficiales adoptados para la formación de los docentes por el MINED y vinculados directamente a las categorías principales, tal como se detallan en la Figura n. ${ }^{\circ} 1$.

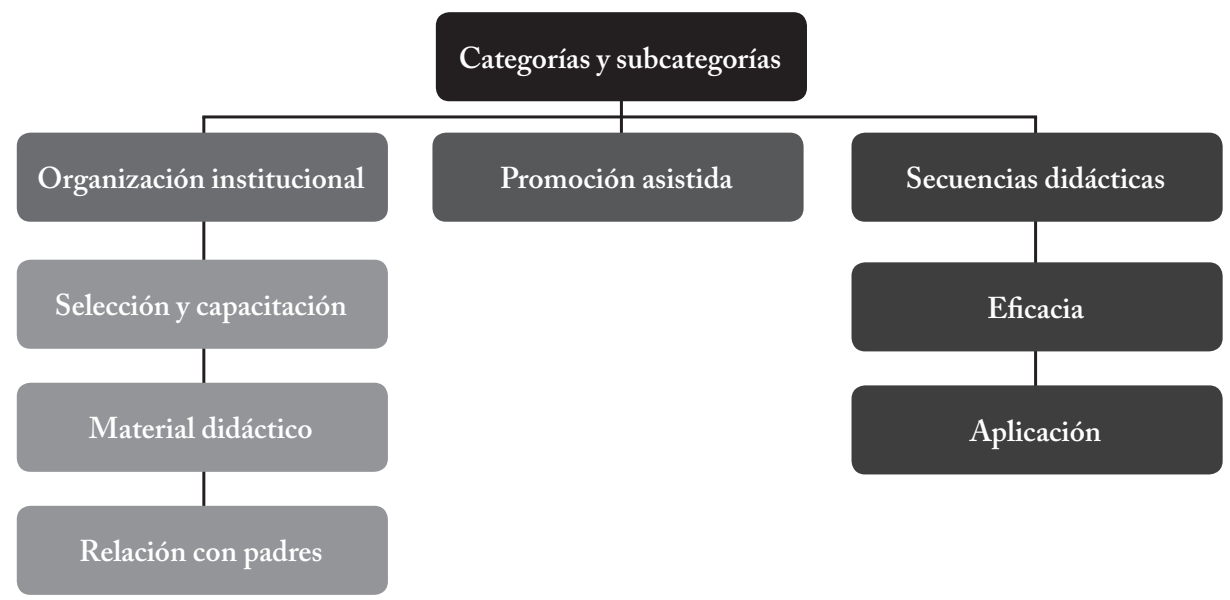

Figura $n .^{\circ}$ 1. Descripción del sistema de categorías y subcategorías. Fuente: elaboración propia. 


\section{Organización institucional}

\section{Selección y capacitación de los docentes}

En la etapa inicial, el proceso de selección y capacitación de los docentes fue informado por los asesores pedagógicos al personal directivo y docente de los centros escolares. La orientación principal fue que el proceso de capacitación sería coordinado a través de la ESMA (Escuela Superior de Maestros) en la aplicación del proyecto piloto: “Todos Pueden Aprender”. Esta fase no demostró problemas operativos en su aplicación desde la perspectiva de los directores y maestros, los cuales, incluso, argumentan que fueron seleccionados por su desenvolvimiento en el ejercicio docente. A continuación se presentan comentarios del personal consultado:

El proceso de selección fue de acuerdo a la cantidad de alumnos que teníamos, y también al desenvolvimiento que tiene cada docente porque -Todos Pueden Aprenderes una estrategia activa, entonces yo siento que por ahí fue donde el director seleccionó el personal...

Bueno, primero el director, y el técnico, nos informaron que iban a dar una capacitación, que iban a echar a andar un proyecto piloto que viene del ESMA. Lo vino a impartir una docente de la universidad de Lenguaje y Matemática...

Además, se preguntó a los participantes su percepción sobre la calidad de la capacitación, $y$, si esta fue suficientemente "convincente" para la aplicación de las secuencias didácticas con los niños y las niñas. Las percepciones en este aspecto indican que en un primer momento, las características activas de las secuencias didácticas fueron de difícil aceptación, en la medida que representa una "novedad" metodológica de enseñanza, principalmente sintéticos o silábicos. Pero que eso no impidió su aplicación en el aula, aunque sí se enfatiza en los problemas metodológicos para ejecutar durante la fase inicial. A continuación el comentario sobre este aspecto:

En un primer momento, como todo eso era nuevo, fuimos a tres capacitaciones, más que todo así yo lo sentí como que era algo nuevo, que estábamos innovando, me sentía (al principio) como que iba tambaleando... no veíamos que se tomaba; por ejemplo una palabra eje para enseñar una consonante pero a medida que veíamos que con la repetición se trabaja en base a textos narrativos, instructivos, los descriptivos; en un texto uno va sacando las rimas, el número de palabras, las palabras cortas, y dije: ¡sí entendí! Pero nada más que con los niños cuesta al principio...

Otro factor detectado es que si bien los procesos de capacitación fueron comprendidos, la necesidad constante de capacitarse para mejorar las prácticas educativas que implica la aplicación de las secuencias didácticas debe ser constante, y en esto no hubo la suficiente coordinación por parte del Ministerio de Educación. Al respecto, se registró una percepción que consideraba que si bien el proyecto es "bonito", hacía falta mayor capacitación. Se presenta a continuación una de las opiniones obtenidas:

Eso (se refiere a la estrategia TPA) para nosotros fue bonito, toda la explicación que nos dieron ahí fue bonito. Pero ya 
venirlo a aplicar aquí, a la práctica es un obstáculo. Por ejemplo: en este año solo dos capacitaciones tuvimos, no hemos tenido más. Es que el Ministerio echó a andar este plan con el personal de las universidades de San Salvador, luego, ya para este año yo creo que no tenían recursos quizás, por tanto trabajo que tienen ellos, nosotros tuvimos hasta una equivocación que fuimos a dar ahí y no nos tocaba... (Profesora del C. E. La Presita, 2015).

\section{Utilidad del material didáctico}

Se evaluó la percepción de los docentes sobre el material didáctico entregado en primera instancia para la aplicación de las secuencias didácticas. Las respuestas indican que, los docentes admiten que les fueron entregados los materiales necesarios como libros y fotocopias de las secuencias didácticas. No obstante, en la medida en que fue trascurriendo el tiempo, ya no hubo seguimiento para la entrega del material complementario, e incluso en algunas ocasiones hasta se entregó de manera desorganizada. A continuación se presenta un comentario sobre el tema:

La segunda secuencia no la tenemos, nos dieron primero la tercera y luego la primera y nosotros así la dimos. Esto lo hemos dado distorsionado. Nosotros desarrollamos lo que nos dieron, pero eso nos dieron, eso desarrollamos...

El acceso a recursos complementarios ha sido posible gracias al acompañamiento de los padres y en buena medida a la intervención del director con fotocopias y papelería. Así lo expresó una de las personas consultadas:
Él(director) nos ha ayudado con la facilitación de unas fotocopia, se requieren sacar bastante para que alcance para todos los niños porque el libro solo trae un ejemplar; entonces, ese ejemplar hay que reproducirlo y el director ha estado accesible para darnos eso; y otra cosa que nos ha accedido es el tiempo, porque cada vez que hay un final de una secuencia nos dan tiempo para que elaboremos el material para la siguiente secuencia...

Existe apoyo institucional en el afán de facilitar los recursos, aunque de manera no planificada o en torno a un proyecto institucional, tal cual lo sugieren los documentos de formación. Es más, el deber administrativo el que motiva a los directores a poder facilitar algunos materiales.

\section{Relación de favorecimiento con los padres de familia}

A nivel institucional, la metodología principal para el establecimiento de las relaciones con los padres de familia ha sido a través de reuniones informativas coordinadas por las autoridades de los centros escolares conforme a la planeación escolar. Pero, principalmente, el involucramiento de los "adultos significativos" en el proceso de aprendizaje de los niños se ha realizado a través de las mediaciones de las docentes cotidianamente, quienes han construido las relaciones favorables con el afán de facilitar el trabajo de acompañamiento, al cual han accedido satisfactoriamente los padres de familia. Y, ha sido tan buena la aceptación y la implicancia de los padres para facilitar el desarrollo de las secuencias didácticas, que estos, han participado en la recolección de recursos necesarios para su ejecución. Los docentes de 
los centros escolares analizados en este estudio, han procurado una introyección en los padres de los beneficios educativos para la implementación de la estrategia educativa. Así opinó una de las personas consultadas:

No fue nada difícil, al contrario, fueron bien accesibles, porque cuando empezamos el proyecto en primer grado, a los niños se les pidió material adicional, entonces la mayoría de padres correspondieron con el material que se les pidió...

Fue fácil (con los padres de familia). En una reunión, yo les dije todo lo que teníamos que hacer y los padres de familia accedieron, porque, incluso hay unos materiales como tapones, semillas, corcholatas, (pelotas) chibolas, semillas de anona, todos esos son materiales que nos han facilitado los padres, botellitas, aros que son los que nosotros hemos necesitado...

La asimilación de las "tareas" era un punto determinante en la aceptación de la estrategia por parte de la comunidad. Las secuencias didácticas que se encuentran en el marco de la propuesta metodológica de la enseñanza activa no son congruentes con las tradicionales tareas de reforzamiento (planas, traspaso de escritos de libros, operaciones), lo cual se esperaba pudiera resultar en críticas a la representación tradicional de "buena enseñanza” del docente, y de la institución. No obstante, según los docentes esto se ha resuelto a través de un proceso de adaptación. Los padres en la medida en que se reiteran las "nuevas" tareas, que implica la implementación de la estrategia, se adecúan y reconocen los beneficios educativos. A continuación una de sus opiniones:

Los padres siempre me han colaborado, si se les informa previamente en una reunión, y normalmente preguntar el por qué ya no se hacen las planas, pero le vuelvo a repetir las tareas, en medida en la que van adaptándose al proceso de la metodología...

En general, la experiencia de los docentes y la sana relación con la mayoría de padres de familia fueron factores claves para vincular a los padres en el proyecto. Pese a un poco de resistencia cultural, se observa que en la medida en que las actividades son informadas y el docente persiste en la aplicación de las secuencias didácticas, los padres van asimilando paulatinamente las ventajas educativas ofrecidas.

\section{La metodología de la Promoción Asistida}

Uno de los ejes fundamentales para la superación del fracaso escolar, especialmente el problema de la repitencia en la propuesta pedagógica de la estrategia, es la implementación de la metodología de "Promoción Asistida". En concreto, se pretendía evaluar dos aspectos a saber: a) la asimilación y aplicación del concepto de la promoción asistida ejecutada por el docente y el seguimiento a los estudiantes con problemas de aprendizaje; y b) la administración institucional para permitir al docente que dé continuidad durante los 3 años que implica el nivel primario.

En el primer aspecto, los resultados indican que los docentes han asimilado el concepto del problema de la repitencia en los niños y niñas, y 
han podido aplicarlo de manera efectiva con los grupos de estudiantes, a excepción de aquellos casos donde los niños presentan problemas de aprendizaje de carácter específico:

Yo le puedo dar el ejemplo, yo tengo niños que en primer grado no me aprendieron a leer y a escribir, pero no los dejé reprobados, sino que en segundo grado traté de buscar estrategias que me ayudaran a nivelarlo y ahí están, aprendieron los “cipotes...” (niños).

¡Vaya! la cuestión es que la promoción es masiva, entonces hay tres años para que el niño pueda aprender, porque ese proyecto de "Todos Podemos Aprender" es de darle continuidad. Si el niño se quedó en una tarea hasta la numero seis, en segundo grado unos los llevan porque el programa así es, de llevar al alumno de primero, segundo, hasta tercero...

Sobre este punto, llama la atención la perspectiva de una docente, la cual comprende el principio de metodología de la Promoción Asistida como promoción automática, que obliga a llevar al niño al siguiente grado sin seguimiento didáctico e institucional. No obstante, considera que esto, en última instancia, debe ser una decisión de los padres de familia, dado que desde su perspectiva, estos deben de decidir:

La Promoción Asistida es la que va pasando, y allá en el otro grado da, bueno y si no va al siguiente grado, o sea; a no dejarlo aplazado $\mathrm{y}$ si lo he aplicado. Ha habido unos que otros, pero como le digo ahí se van. Pero me ha pasado que un papá que reconoce que su hijo no sabe leer no está de acuerdo con pasarlo a segundo grado. Verá, algunos padres sí están de acuerdo, y otros no. Dicen que no están de acuerdo con la Promoción Asistida. Si el padre decide que su hijo no está apto para pasar a segundo y que se deje, ahí se deja...

La representación anterior definitivamente no está en función de la metodología por dos razones: la decisión de aprobar o no aprobar a un niño al grado superior no puede estar en manos de los padres cuya función solo debe ser de acompañamiento del proceso pedagógico y; porque el diseño de la metodología trata de un seguimiento a los niños con problemas de aprendizaje a través de maneras activas de enseñar. La percepción de la docente indica que no hay una distinción clara sobre la promoción automática y la Promoción Asistida.

El segundo aspecto tenía como interés abordar la disposición de los directores para favorecer el desarrollo organizativo de la metodología de la Promoción Asistida. En este sentido, se encontró que en tres de los cuatro centros escolares, en efecto, los docentes que iniciaron en primer grado desde la implementación de la estrategia estaban atendiendo el tercer grado. La excepción esgrimió sus razones en que solo pudieron dar cumplimiento con el encuadre normativo hasta segundo grado, por motivos de la cualificación del recurso humano. Desde la perspectiva del director no "cualquiera" puede ser docente de primer grado, y por consiguiente tuvo que suspender el proceso:

(...) como institución no llevamos el servicio hasta tercer grado lo llevamos hasta segundo... porque no tenemos compañero que sobresalga para trabajar con primero; 
recuerda que ese es el grado más bonito, y tiene que estar preparado el maestro para dar ese grado y los que íbamos a traer que hoy están en tercero, no iban a rendir lo suficiente con primer grado por falta de competencias. Tuve que tomar la decisión de designar en a primero, la que iba para tercer grado porque la persona no iba a responder al primer grado...

Problemas como el descrito con anterioridad ocurren con frecuencia en los centros escolares. Sin embargo, a la existencia de situaciones especiales, se puede valorar que existe voluntad administrativa por ir consolidando el encuadre normativo que implica la metodología de la Promoción Asistida. Y esto radica, principalmente, en la credibilidad que adquiera con su efectividad como alternativa pedagógica para disminuir el fracaso escolar, lo cual ya ha sido observado por los docentes y los directores, en tanto ven al educando en un proceso que puede alcanzar el éxito si se asimila el principio de asistencia que implica la promoción.

\section{Las secuencias didácticas}

\section{Eficacia de las secuencias didácticas}

Una de las innovaciones que propone la estrategia educativa “Todos Pueden Aprender" es la planeación de la secuencia didáctica a través de propuestas cognoscitivas que se presentan a los estudiantes como tareas de trabajo en el salón de clases. Cabe decir que la inclusión de las secuencias didácticas ha sido de manera complementaria a la planificación normal del docente, y se ejecuta por periodos coordinados por el asesor pedagógico y el director. Por lo que se debe tener en cuenta que dichas secuencias son asumidas por los docentes como recursos auxiliares para el desarrollo de los contenidos y el alcance de los indicadores de logro.

Tomando en cuenta lo anterior, se preguntó a los docentes, si consideraban efectiva la secuencia didáctica propuestaporla estrategiaeducativacomo un método eficaz para estimular el aprendizaje, y facilitar el desarrollo de competencias en las áreas de lenguaje y matemáticas. La percepción al respecto es la siguiente:

Sí se desarrollan. El niño a veces no sabe leer pero reconoce por medio de figuras, hay poemas que memorizan. La expresión oral, mire para enseñar a leer y escribir para mí es un complemento porque más que todo aquellos que están deficientes les ayuda más, ellos ya están adaptándose y no lo hemos dejado del todo, lo hemos llevado a la par...

Por la recurrencia es que la propuesta didáctica de la estrategia educativa "Todos Pueden Aprender" es sumamente positiva para el aprendizaje de los estudiantes. Las docentes han observado que las cualidades activas, y la integración de elementos propios del entorno favorecen el desarrollo cognitivo del estudiante.

\section{Problemas de las secuencias didácticas}

También se indagó con los docentes, si en el proceso de implementación habían tenido dificultades en la comprensión y posterior ejecución de las secuencias didácticas y su percepción de los resultados fue la siguiente: 
Únicamente, el problema que siento es que en la aplicación de la secuencia, lo único es la inasistencia, es algo que en vez de avanzar el niño retrocede, porque perdió la clase participativa con sus compañeros, la reacción del estudiante: excelente porque el niño se nota despierto, se nota activo...

Se puede observar que los principales problemas tienen dos orígenes: por una parte aquellos derivados de la deserción temporal o permanente de los niños y niñas, la cual indudablemente repercute debido al carácter in situ de la aplicación de la estrategia en el salón de clases; y por otra parte, la comprensión de la propuesta didáctica, para su aplicación. No obstante, aparece como positivo el hecho de que uno de los medios principales para subsanar dicha problemática es el trabajo colaborativo entre docentes. Cabe decir, que los maestros considerados en este estudio, pese a que tienen más veinte de años de experiencia, están muy acostumbrados al método silábico de alfabetización inicial. Por lo que la aplicación de estrategias educativas con enfoques participativos es en alguna medida difícil de incluir en el ejercicio docente. Una supervisión adecuada aunada a la incentivación de una actitud positiva al docente puede ejercer un efecto actitudinal que motive al docente a la constante actualización para la aplicación de la metodología.

\section{Discusión de los resultados}

Los resultados de la evaluación indican la existencia de elementos sumamente positivos en términos institucionales y metodológicos, y otros que son imprescindibles mejorar para volver más efectiva la implementación del encuadre normativo que necesita la estrategia educativa
TPA para ser funcional. Específicamente los aspectos vinculados a la organización que implica en la comunidad educativa.

En la evaluación del área institucional se encontró que los docentes están conscientes de que han sido seleccionados por sus competencias y experiencia en el trabajo con niños y niñas de educación básica, lo cual estimula la confianza para poder encarar el reto de la aplicación de la propuesta didáctica que implica la estrategia educativa. No obstante, en términos de la formación requerida, si bien la idea o noción central se comprendió durante las capacitaciones iniciales, la falta de continuidad durante este proceso y la dificultad para la obtención de las secuencias didácticas (sobre todo del tercer nivel), desmotivó y repercutió en buena medida en la calidad de la aplicación en el aula de la estrategia.

Otro hallazgo importante es que la socialización de la estrategia por parte del director hacia la comunidad no ha adquirido la suficiente importancia en el marco de la implementación de la estrategia. De hecho no se observó para efectos de este estudio, del lanzamiento del Programa con la suficiente difusión e importancia que implica. Han sido los docentes en la práctica, los que se han encargado de transmitir a los padres de familia el desarrollo de las secuencias didácticas y algunos elementos necesarios para la aplicación de las mismas.

E1 seguimiento de los directores a la implantación de la estrategia educativa fue más de proporción de insumos en papelería y gestión de libros. Y la parte de la evaluación sobre el aprendizaje estuvo bajo la responsabilidad 
de los asesores técnicos pedagógicos. En este sentido, en el discurso de los docentes aparece muy débil el acompañamiento en el área metodológica de la aplicación de la estrategia. Para los docentes es más importante recibir capacitaciones, que el acompañamiento de los técnicos locales.

En relación con la evaluación de la implementación de la metodología de la promoción asistida, se puede subrayar que los docentes manifestaron, en sus narraciones, los conocimiento sobre los principios teóricoconceptuales de la promoción directa y asistida para la superación del fracaso escolar. Sin embargo, se encontraron problemas de sustentación de la metodología en situaciones que algunos denominaron casos "especiales" de niños con problemas de aprendizaje, y/o por las decisiones de los padres de familia de hacer repetir el año escolar a los niños. Dicha situación corresponde a que si bien se tiene conocimiento respecto a los principios, no se ha impregnado, en algunos casos, del principio pedagógico de la metodología respecto a las consecuencias de la repitencia del año escolar y su relación con la superación del fracaso escolar como parte de la inclusión del niño a la sociedad.

\section{Recomendaciones}

Tomando en cuenta la discusión de los resultados del estudio, se derivan las siguientes recomendaciones para la mejora de la implementación de la estrategia educativa TPA:

a. Es preciso mejorar la gestión escolar de la estrategia educativa por parte de las direcciones de los centros escolares. Principalmente, en el lanzamiento del proyecto hacia los padres de familia, el seguimiento de la implementación metodológica de las secuencias didácticas y la provisión de recursos didácticos con la finalidad de facilitar el encuadre normativo que demanda la organización institucional.

b. La función de asesoría de los asistentes técnico-pedagógicos debe orientarse a fortalecer en el docente la planeación, ejecución y evaluación de las secuencias didácticas a nivel de fundamentación pedagógica y metodológica. Éstos deben volverse protagonistas ante los problemas cotidianos de los docentes por las características activas de la estrategia, que es donde se presentan las mayores dificultades.

c. Se recomienda a los maestros mantener el criterio pedagógico que da lugar a la promoción asistida y directa, independientemente de la exigencias de otros actores involucrados, mediante el diálogo conciliador y convincente de los beneficios de no irrumpir la trayectoria educativa del niño ante los demás miembros de la comunidad educativa

d. Es preciso que los docentes y las autoridades a nivel institucional reconozcan las deficiencias en el manejo de contenidos, específicamente en el área de matemáticas. Para ello se pueden emplear capacitaciones específicas sobre dichos temas o el involucramiento de otros actores especializados que permitan superar las mencionadas deficiencias, en función de que estas no afecten el rendimiento académico de los estudiantes. 


\section{Referencias}

Asociación Civil Educación para Todos. (2015). Educacion para Todos. Recuperado el 15 de Mayo de 2015, de http://www.educacionparatodos. org.ar/aept/todospuedenaprender.asp

Asociación Educacion Para Todos. (s.f.). Innovemos. Recuperado el 17 de Noviembre de 2015, de www.innovemosdoc.cl/diversidad_ equidad/programa/TPA.pdf

Bausela Herreras, E. (2004). Metodología de Investigación Evaluativa. Indivisa. Boletin de Estudios e Investigación, 183-191.

González Moteagudo, J. (2001). El paradigma interpretativo en la investigación social y educativa: nuevas respuestas para viejos interrogantes. Revista Ciencias de la Educación, 227-246.
Ministerio de Educación. (2010). Política de Educación inlcusiva. San Salvador: Autor.

Ministerio de Educación de El Salvador (MINED). (2014). "Revisión Nacional 2015 de la Educación para Todos: El Salvador.”. Obtenido de http://unesdoc.unesco.org/ images/0023/002300/230031S.pdf

UNESCO. (2014). Enseñanza y Aprendizaje. Lograr la Calidad para todos. Francia: UNESCO.

UNICEF. (2007). Propuestas para superar el Fracaso Escolar. Obtenido de http://files.unicef. org/argentina/spanish/PROPUESTA_web.pdf

UNICEF. (2007). Todos Pueden Aprender Lengua y Matemática en el Primer Ciclo. Buenos Aires: UNICEF. 